\title{
Scales of Linear Baroclinic Instability and Macroturbulence in Dry Atmospheres
}

\author{
TIMOTHY M. MERLIS AND TAPIO SCHNEIDER
}

California Institute of Technology, Pasadena, California

(Manuscript received 11 July 2008, in final form 20 November 2008)

\begin{abstract}
Linear stability analyses are performed on a wide range of mean flows simulated with a dry idealized general circulation model. The zonal length scale of the linearly most unstable waves is similar to the Rossby radius. It is also similar to the energy-containing zonal length scale in statistically steady states of corresponding nonlinear simulations. The meridional length scale of the linearly most unstable waves is generally smaller than the energy-containing meridional length scale in the corresponding nonlinear simulations. The growth rate of the most unstable waves increases with increasing Eady growth rate, but the scaling relationship is not linear in general. The available potential energy and barotropic and baroclinic kinetic energies of the linearly most unstable waves scale linearly with each other, with similar partitionings among the energy forms as in the corresponding nonlinear simulations. These results show that the mean flows in the nonlinear simulations are baroclinically unstable, yet there is no substantial inverse cascade of barotropic eddy kinetic energy from the baroclinic generation scale to larger scales, even in strongly unstable flows. Some aspects of the nonlinear simulations, such as partitionings among eddy energies, can be understood on the basis of linear stability analyses; for other aspects, such as the structure of heat and momentum fluxes, nonlinear modifications of the waves are important.
\end{abstract}

\section{Introduction}

In Earth's atmosphere, scales of the energy-containing eddies are similar to those of the linearly most unstable baroclinic waves. The energy-containing length scale (spherical wavenumber $\sim 8$ ) is similar to the zonal length scale of the most unstable waves, and the eddy turnover time ( $\sim 3-5$ days) is similar to their inverse growth rate (e.g., Shepherd 1987b; Simmons and Hoskins 1976; Valdes and Hoskins 1988). Analysis of observations shows that eddy-mean flow interactions, not nonlinear eddy-eddy interactions, dominate the spectral energy transfers; there is no extended wavenumber range of nonlinear (transient) eddy-eddy interactions that could give rise to an inverse energy cascade and an upscale transfer of eddy energy from the scale of generation by baroclinic instability to significantly larger scales (Shepherd 1987a,b). Theory and simulations with an idealized dry general circulation model (GCM) suggest this agreement of linear wave scales and non-

Corresponding author address: Timothy Merlis, 1200 East California Blvd., MC 100-23, California Institute of Technology, Pasadena, CA 91125.

E-mail: tmerlis@caltech.edu linear eddy scales does not occur by chance but, rather, results because macroturbulence modifies the atmospheric thermal structure such that nonlinear interactions among turbulent eddies are weak (Schneider and Walker 2006). Nonetheless, while the nonlinear eddyeddy interactions that cause differences between linear wave scales and nonlinear eddy scales may be weak, they are not absent altogether. For example, meridional momentum fluxes associated with linear waves are generally confined to a narrower latitude band than those associated with fully developed nonlinear eddies, indicating that linear waves have smaller meridional scales and/or do not propagate meridionally as effectively as the nonlinear eddies (Gall 1976a,b; Simmons and Hoskins 1976, 1978; Edmon et al. 1980; O'Gorman and Schneider 2007).

As the basis for a systematic study of differences between linear waves and fully developed nonlinear eddies, here we present linear stability analyses of a wide range of atmospheric mean flows simulated with an idealized dry GCM. The mean flows are a subset of those whose fully developed nonlinear dynamics were examined in Walker and Schneider (2006), Schneider and Walker (2006, 2008, hereafter SW06 and SW08, respectively) and Schneider and Merlis (2009, unpublished 
manuscript, hereafter SMER), allowing us to compare properties of linear waves with theoretical predictions, on the one hand, and with properties of nonlinear eddies on the other. The use of a wide range of simulations enables us to make such comparisons systematically. For example, we are able to compare mean-field estimates for scales of linear waves based on idealized quasigeostrophic models with scales of linear waves on a wide range of complex mean flows in a primitive equation model, allowing us to determine empirical constants in scaling relations. The systematic comparison of linear waves and nonlinear eddies in dry atmospheres is a necessary prerequisite for further studies in atmospheric macroturbulence that may attempt to develop turbulence closures (e.g., based on weakly nonlinear models) or add neglected physical processes (e.g., latent heat release in phase changes of water). In this paper, we (i) show that the mean flows whose nonlinear dynamics were examined in SW06, SW08, and SMER are indeed baroclinically unstable, (ii) demonstrate that mean-field estimates such as the Rossby radius and Eady growth rate account for properties of linear waves such as their zonal length scale and growth rate (albeit not always precisely), and (iii) examine limitations of linear stability analysis in accounting for properties of nonlinear eddies.

\section{Idealized GCM and linear stability analyses}

\section{a. Model description}

We performed linear stability analyses of the simulated mean flows described in SW06, SW08, and SMER. The GCM with which the flows are simulated is based on the spectral dynamical core of the Geophysical Fluid Dynamics Laboratory's Flexible Modeling System. It integrates the primitive equations on the sphere, discretized with the spectral transform method in the horizontal (resolution from T42 to T127 for different simulations), and with 30 unevenly spaced sigma coordinate levels in the vertical. Forcing and dissipation in the nonlinear simulations consist of Newtonian relaxation of temperatures as a representation of radiation, a turbulent boundary layer scheme (roughness length $5 \mathrm{~cm}$ ) acting in the lowest $2.5 \mathrm{~km}$ of the model atmosphere (Smagorinsky et al. 1965), and $\nabla^{8}$ hyperviscosity acting on vorticity, divergence, and temperature. A quasi-equilibrium dry convection scheme relaxes temperatures to a profile with convective lapse rate $\gamma \Gamma_{d}$, where $\Gamma_{d}$ is the dry-adiabatic lapse rate $\Gamma_{d}=g / c_{p}$, whenever a column is statically less stable than a column with the convective lapse rate. If $\gamma<1$, the convection scheme mimics some of the stabilizing effect that latent heat release has on the thermal structure of the atmosphere (details can be found in appendix B of SW06).

The Newtonian relaxation is toward the radiative equilibrium of a semigray atmosphere (transparent to shortwave radiation and gray to longwave radiation), with a spatially varying time scale. The surface temperature in radiative equilibrium varies with latitude $\phi$ as (SW06)

$$
T_{s}^{e}(\phi)=\tilde{T}_{s}^{e}+\Delta_{h} \cos ^{2} \phi .
$$

The pole-equator surface temperature contrast $\Delta_{h}$ is one of the parameters varied to obtain different mean flows; the polar temperature $\tilde{T}_{s}^{e}=260 \mathrm{~K}$ is constant in all simulations. The vertical structure of the radiative equilibrium temperature is given by

$$
T^{e}(\phi, p)=T_{t}^{e}\left[1+d_{0}(\phi)\left(\frac{p}{p_{0}}\right)^{\alpha}\right]^{1 / 4}
$$

with constant skin temperature $T_{t}^{e}=200 \mathrm{~K}$ at the top of the atmosphere, reference pressure $p_{0}=1000 \mathrm{hPa}$, and exponent $\alpha=3.5$. The optical depth

$$
d_{0}(\phi)=\left[\frac{T_{s}^{e}(\phi)}{T_{t}^{e}}\right]^{4}-1
$$

is chosen so that the radiative equilibrium temperature makes a continuous transition from the constant skin temperature $T_{t}^{e}$ to the surface temperature $T_{s}^{e}(\phi)$ (Schneider 2004).

The Newtonian relaxation time $\tau_{r}$ varies with latitude and pressure according to

$$
\tau_{r}^{-1}(\phi, \sigma)=\tau_{i}^{-1}+\left(\tau_{s}^{-1}-\tau_{i}^{-1}\right) \max \left(0, \frac{\sigma-\sigma_{b}}{1-\sigma_{b}}\right) \cos ^{8} \phi,
$$

where $\sigma=p / p_{\mathrm{s}}$ with surface pressure $p_{\mathrm{s}}$ and $\sigma_{b}=0.85$ (Schneider 2004). The time constants $\tau_{i}$ and $\tau_{\mathrm{s}}$ allow different relaxation times in the interior atmosphere and near the surface in low latitudes.

\section{b. Series of simulations}

Several series of nonlinear, forced-dissipative simulations have been performed with the idealized GCM. Here we describe the subset of simulations for which we performed linear stability analyses. In one set of simulation series, we set the convective rescaling parameter to values $\gamma=(0.6,0.7,0.8,0.9)$ and, for $\gamma=0.7$, we set the planet rotation rate $\Omega$ and radius $a$ to Earth values $\Omega_{e}$ and $a_{e}$ and values two and four times larger. In these simulations, the Newtonian relaxation times are $\tau_{i}=50$ 
TABLE 1 . Values of $\Delta_{h}$ for each of the series of simulations for which a linear stability analysis is presented.

\begin{tabular}{ll}
\hline \multicolumn{1}{c}{ Series } & \multicolumn{1}{c}{$\Delta_{h}(\mathrm{~K})$} \\
\hline$\gamma=(0.6,0.7,0.8,0.9)$ & $22.5,30,45,60, \ldots, 180,210, \ldots, 300,360$ \\
$2 \Omega_{e}$ & $22.5,30,45,60, \ldots, 180,210, \ldots, 300,360$ \\
$4 \Omega_{e}$ & $30,60,90, \ldots, 300,360$ \\
$2 a_{e}$ & $45,60, \ldots, 180,210, \ldots, 300,360$ \\
$4 a_{e}$ & $90,120, \ldots, 300,360$ \\
$\tau=(12.5,25,50,100,200)$ days & $60,90, \ldots, 300,330,360$ \\
\hline
\end{tabular}

days in the interior atmosphere and $\tau_{\mathrm{s}}=7$ days at the surface in low latitudes. In another set of simulation series, we used a spatially constant Newtonian relaxation time $\left(\tau_{s}=\tau_{i}=\tau\right)$ and set it to values $\tau=(12.5,25$, $50,100,200)$ days. The hyperviscosity is chosen such that the smallest resolved scale is damped on a time scale of $12 \mathrm{~h}$ in all simulations, except in the series with constant relaxation times in which the damping time scale is $2.4 \mathrm{~h}$.

For each setting of $\gamma, a, \Omega$, and $\tau$, the pole-equator radiative-equilibrium temperature contrast $\Delta_{h}$ is varied over a wide range (Table 1). The lowest radiativeequilibrium temperature contrasts are omitted for the larger planet radii to keep the lowest value of $\Delta_{h} / a$, a measure of the baroclinicity of the radiative-equilibrium state, the same for the different planet radii.

For this study, we have omitted some simulations presented in SW06, SW08, and SMER. Those papers include 13 simulations with lower radiative-equilibrium temperature contrasts $\Delta_{h}$. The mean flows of at least 6 of the 13 are linearly unstable but, because the growth rates are small, the unstable waves may not be well resolved by our linearization method; the remainder may or may not be linearly unstable. Also, a series of simulations with the convection scheme relaxing temperature profiles toward dry adiabats $(\gamma=1.0)$ is omitted because the mean thermal stratifications are statically unstable in low latitudes, which prevents an accurate determination of the most unstable waves. Finally, a series of simulations with lower roughness length has been omitted. In total, we present the results of linear stability analyses for 175 of the 223 mean flows of the nonlinear simulations.

\section{c. Linear stability analysis}

Linear waves are calculated by perturbing one zonal wavenumber at a time, only allowing the spectral coefficients of the perturbed zonal wavenumber to evolve in time, and keeping the amplitude of the growing wave small by rescaling the wave when it reaches a threshold amplitude. For example, the time tendency of temperature has nonlinear advection terms such as the meridional advection of temperature

$$
\begin{aligned}
\frac{\partial T}{\partial t} & =-v \frac{\partial T}{\partial y}+\cdots, \\
& =-\bar{v} \frac{\partial \bar{T}}{\partial y}-\bar{v} \frac{\partial T^{\prime}}{\partial y}-v^{\prime} \frac{\partial \bar{T}}{\partial y}-v^{\prime} \frac{\partial T^{\prime}}{\partial y}+\cdots,
\end{aligned}
$$

which is replaced with

$$
\frac{\partial T^{\prime}}{\partial t}=-\bar{v} \frac{\partial T^{\prime}}{\partial y}-v^{\prime} \frac{\partial \bar{T}}{\partial y}+\cdots
$$

where bars indicate zonal means and primes deviations therefrom. (The equations are written here in Cartesian geometry for simplicity, but the GCM has spherical geometry.) The linear stability calculation is integrated for 33 days, and growth rates are determined from the last 6 days of the integration; further details of the initialization procedure, time evolution, and sensitivities to parameters are discussed in appendix B.

The turbulent boundary layer scheme in the nonlinear simulations is replaced with Rayleigh drag for the linear calculations (cf. Hall and Sardeshmukh 1998; Held and Suarez 1994). The Rayleigh drag acts on the horizontal velocities with a coefficient $k_{R}$ that decreases linearly in the model's sigma coordinate from a maximum of $k_{0}=2$ day $^{-1}$ (i.e., a time scale of half a day) at the surface to zero at $\sigma_{b}=0.85$ and above:

$$
k_{R}=k_{0} \max \left(0, \frac{\sigma-\sigma_{b}}{1-\sigma_{b}}\right) .
$$

The Rayleigh drag coefficient $k_{0}$ is chosen to be approximately equal to the drag coefficient inferred from the nonlinear simulations if one assumes the zonal momentum is in Ekman balance in the extratropical boundary layer; it is the same in all linear calculations.

In the linear analyses, we use the same Newtonian relaxation of temperatures as in the nonlinear simulations. The sensitivity of the linear waves to the diabatic forcing and Rayleigh drag time scale is discussed in appendix B. Because the zonal mean is held fixed, the mechanical and thermal forcing and hyperviscosity in the linear stability calculations act exclusively on the waves. 


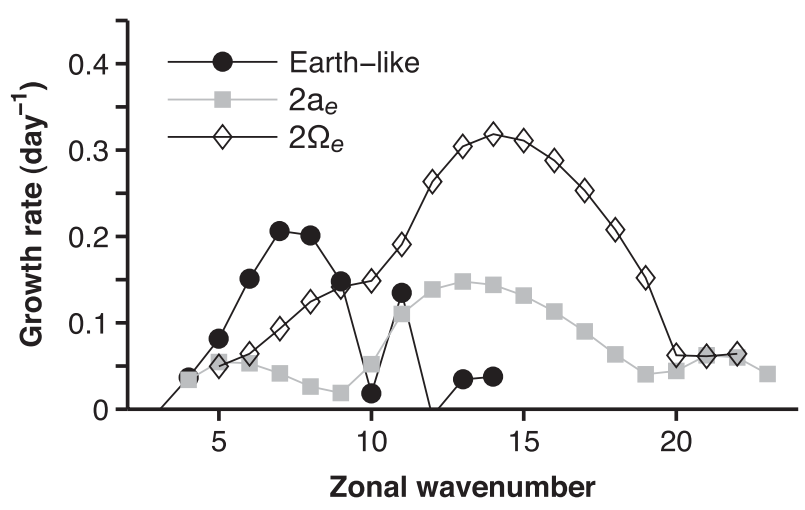

FIG. 1. Growth rate vs wavenumber of an Earth-like mean flow $\left(\Delta_{h}=120 \mathrm{~K}, \gamma=0.7\right)$ and for mean flows with the same radiation and convection parameters but doubled radius or rotation rate.

The convection scheme is not used for our linear stability analysis as convection is a finite-amplitude effect. However, the convection scheme indirectly enters the analysis through its impact on the mean flows of the nonlinear simulations about which we linearize.

For the time- and zonal-mean flows in statistically steady states of the nonlinear simulations, we determined growth rates of linear waves for several zonal wavenumbers (Fig. 1). Fluxes of heat and momentum associated with the linearly most unstable waves (Figs. 2a,c) have smaller meridional extent than those associated with fully developed nonlinear eddies (Figs. 2b,d). These differences in the structure of eddy fluxes occur in all simulations. Also, the eddy momentum flux divergence has a near-surface maximum that is not present in the fully developed nonlinear flow. This is consistent with earlier studies (e.g., Gall 1976a; Simmons and Hoskins 1976). The magnitudes of the potential temperature and momentum fluxes of the linear waves obey the same scaling laws presented in SW08 for nonlinear eddies, but with different constants of proportionality because of larger correlation coefficients between fluctuating flow fields in the linear waves.

\section{Eddy length scales}

The Rossby radius is a mean-field estimate of the length of the linearly most unstable baroclinic wave. We define the Rossby radius as

$$
L_{R}=c_{R} \frac{N_{p}\left(\bar{p}_{s}-\bar{p}_{t}\right)}{f},
$$

where $c_{R}$ is an empirical order-one constant, $\bar{p}_{s}$ is the mean surface pressure, $\bar{p}_{t}$ is the mean tropopause pressure, $f$ is the Coriolis parameter, and $N_{p}^{2}=-\left(\bar{\rho}_{s} \bar{\theta}_{s}\right)^{-1} \bar{\partial}_{p} \theta$ is a static stability measure, evaluated near the surface. ${ }^{1}$ Using near-surface averages is empirically appropriate as the linear waves have large amplitude in the lower troposphere (see Fig. 2).

We express length scales $L$ in terms of zonal wavenumbers $m=a \cos \phi / L$. Our focus is on zonal wavenumbers because this is the quantity that we control when performing the linear stability analysis (see appendix B for details). The meridional scale of the most unstable linear waves may decouple from the zonal scale either by filling the domain as in, for example, the Eady problem (e.g., Vallis 2006, p. 274) or by being constrained by the structure of the mean flow. Consequently, the growing linear waves generally are not isotropic, unlike fully developed nonlinear eddies [see Held (1999) for a discussion of these points].

The first row of Fig. 3 shows that the Rossby radius, with empirical constant $c_{R}=0.8$, approximately accounts for the zonal length scale of the linearly most unstable waves. In Fig. 3, the Rossby radius is evaluated at the latitude of maximum near-surface $(\sigma=0.84)$ eddy flux of potential temperature $\overline{v^{\prime} \theta^{\prime}} \cos (\phi)$ (hereafter, "potential temperature flux") in the nonlinear simulations. This maximum generally occurs in the baroclinic zone and jet closest to the equator if there are several jets in each hemisphere. Evaluating the Rossby radius at the latitude of the maximum potential temperature flux of the linear waves gives similar results.

The linearly most unstable waves generally have largest amplitude near the center or somewhat poleward of the baroclinic zone closest to the equator [as in Valdes and Hoskins (1988)]. There are a few simulations with multiple baroclinic zones for which the most unstable waves have largest amplitude in baroclinic zones in higher latitudes. Leaving those aside, the first row of Fig. 3 demonstrates that, over a wide range of mean flows simulated in a primitive equation model, the local Rossby radius in the baroclinic zone closest to the equator accounts for the length scale of the linearly most unstable waves. However, this does not imply that the local Rossby radius predicts the linearly most unstable length scale latitude by latitude. The initial value problem technique that we use for computing linear waves does not allow one to determine the most unstable waves as a function of latitude.

It was shown in SW06 and SMER that the energycontaining spherical wavenumber is similar to the Rossby wavenumber over the range of simulations. An exception

\footnotetext{
${ }^{1}$ This formulation is similar to that in SW06, though we have omitted the supercriticality factor that appeared there. Including the supercriticality factor does not significantly affect Fig. 3.
} 

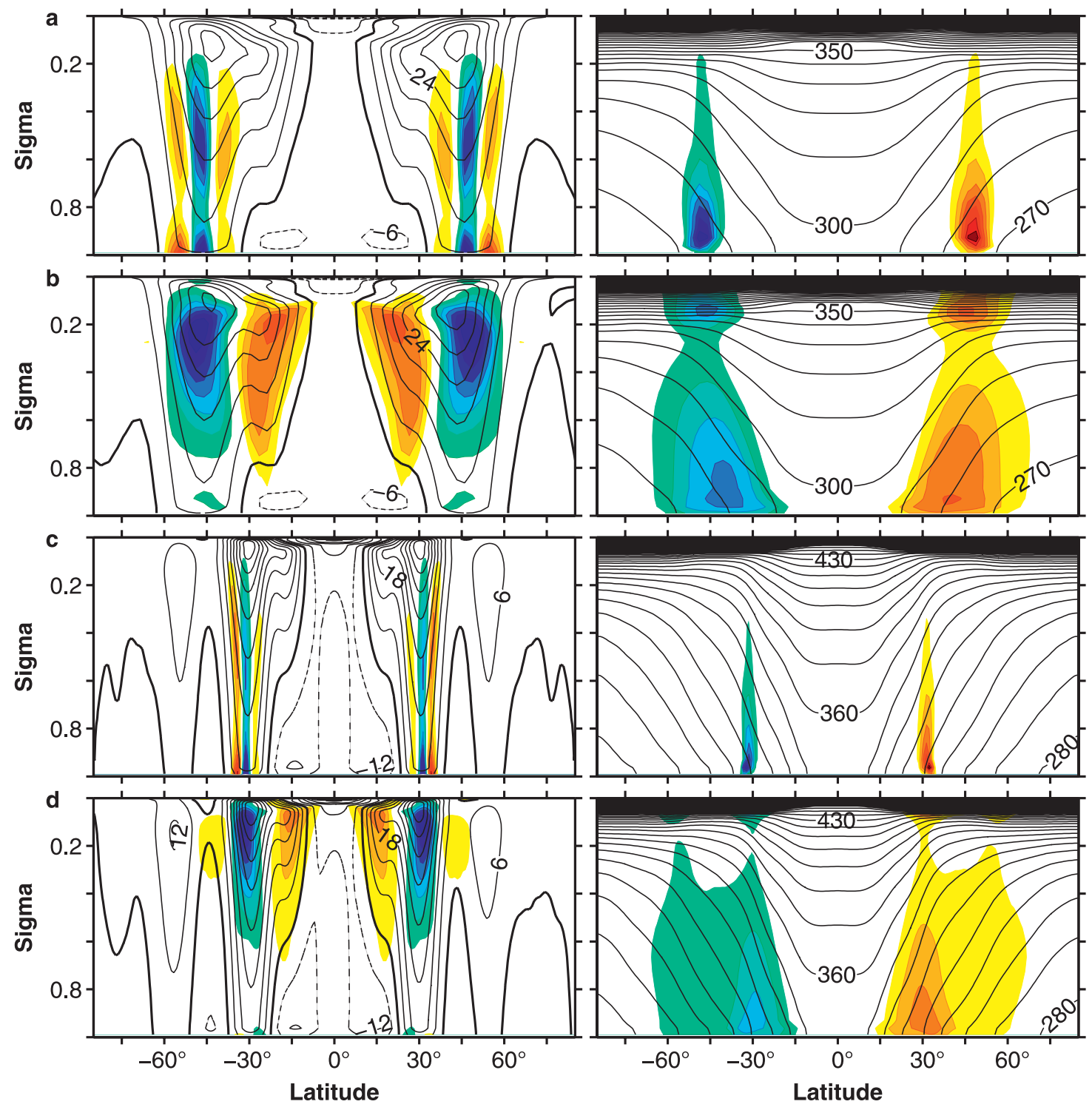

FIG. 2. (left) Mean zonal wind (contours) and eddy momentum flux divergence (colors); (right) mean potential temperature (contours) and eddy potential temperature flux (colors). (a) Eddy fluxes associated with the most unstable wave of a simulation with Earth radius and rotation rate and $\Delta_{h}=90 \mathrm{~K}, \gamma=0.7$. (b) Eddy fluxes from the nonlinear simulation corresponding to (a) with contour interval $4.1 \times 10^{-6} \mathrm{~m} \mathrm{~s}^{-2}$ for the eddy momentum flux divergence and $5.4 \mathrm{~K} \mathrm{~m} \mathrm{~s}^{-1}$ for the potential temperature flux. (c) Eddy fluxes associated with the most unstable wave of a simulation with $\Omega=4 \Omega_{e}, \Delta_{h}=180 \mathrm{~K}$, and $\gamma=0.7$. (d) Eddy fluxes from the nonlinear simulation corresponding to (c) with contour interval $1.6 \times 10^{-5} \mathrm{~m} \mathrm{~s}^{-2}$ for the eddy momentum flux divergence and $11.6 \mathrm{~K} \mathrm{~m} \mathrm{~s}^{-1}$ for the potential temperature flux. Note that (b) and (d) are adapted from Fig. 1 in SW08. Amplitudes of linear eddy fluxes are arbitrary; however, the color scale is chosen to show eight contours of eddy momentum flux divergence and of eddy potential temperature flux for linear waves and nonlinear eddies. The slight differences in the mean fields are due to the hemispheric averaging performed prior to the linear stability analyses.

occurred when the Rossby wavenumber became smaller than the energy-containing wavenumber at large eddy lengths, which SW06 interpreted as a domain-size limitation for the eddy length. Here, we are comparing zonal eddy scales, so we revisit the relationship between the Rossby radius and the energy-containing zonal eddy length scale of the nonlinear simulations.
The second row of Fig. 3 shows that the energycontaining zonal wavenumber is close to the Rossby wavenumber over the entire range of simulations. The scaling also holds at low wavenumbers (large length scales) where the Rossby wavenumber became smaller than the energy-containing spherical wavenumber in the nonlinear simulations. 

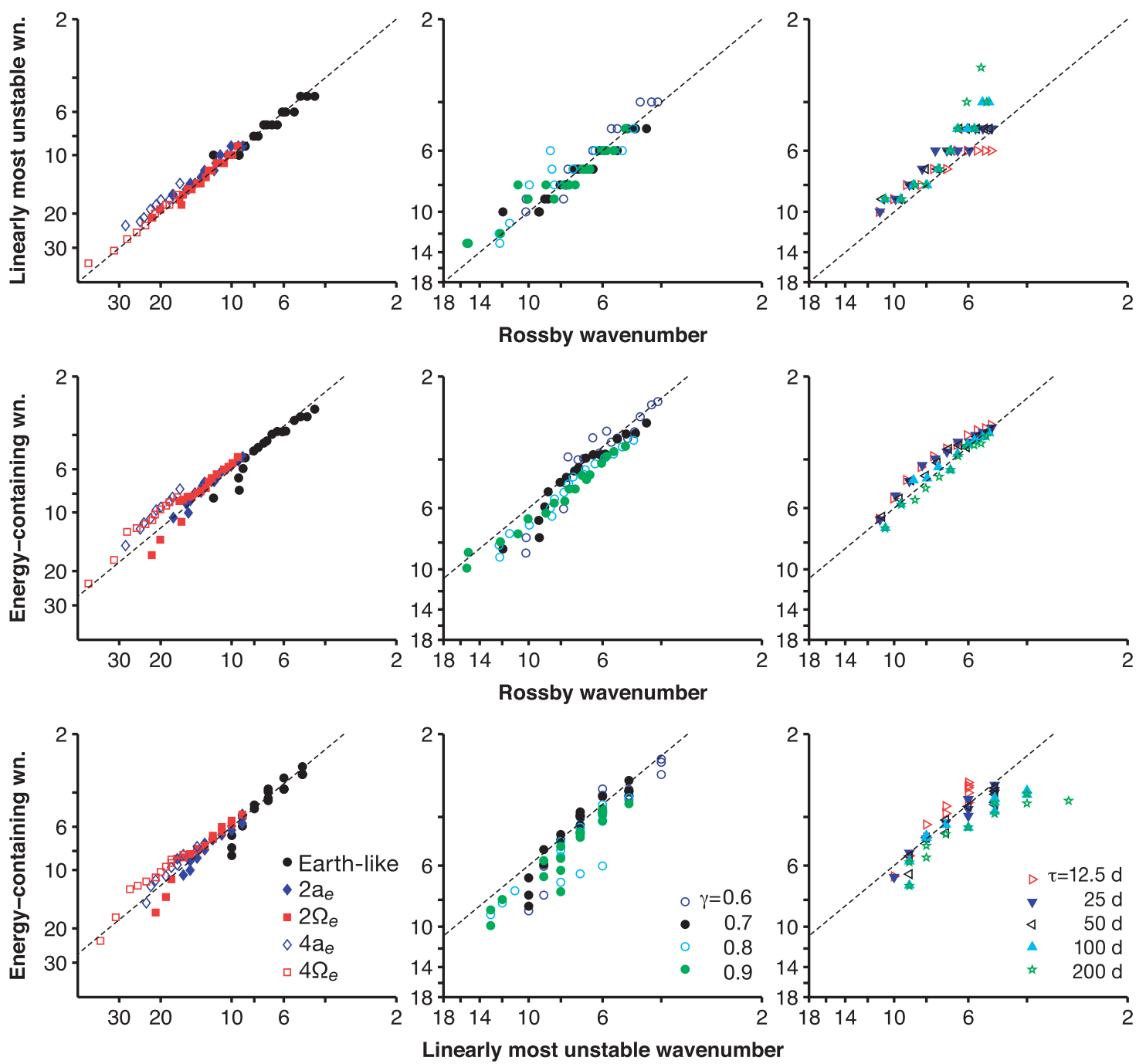

FIG. 3. (top row) Linearly most unstable wavenumber vs Rossby wavenumber with dashed line showing $m_{l}=m_{R}$; (middle row) energy-containing wavenumber vs Rossby wavenumber with dashed line showing $m_{e}=0.6 m_{R}$; (bottom row) energycontaining wavenumber vs linearly most unstable wavenumber with dashed line showing $m_{e}=0.6 m_{l}$. (left column) Simulations with varying radius and rotation rate; (middle column) simulations with varying convective lapse rate; (right column) simulations with varying relaxation time scale. The axes are logarithmic.

The third row of Fig. 3 shows that the energy-containing wavenumber of the nonlinear simulations, evaluated at the latitude of maximum potential temperature flux in the nonlinear simulations, is similar to the wavenumber of the linearly most unstable wave. The dashed lines in these panels have slopes of 0.6: the most unstable wavenumber is generally larger than the energycontaining wavenumber. In terms of eddy lengths, the length scale of the most unstable wave is about $40 \%$ smaller than the typical eddy length of the corresponding nonlinear simulation. Unlike in the analyses of SW06, there are no free parameters in the relation between these length scales, demonstrating unambiguously that the length scale of the most unstable wave and the energy-containing zonal length scale in the nonlinear simulations differ only by an $O(1)$ factor.

One degree of freedom is the latitude at which the energycontaining wavenumber is evaluated. Using the latitude of maximum potential temperature flux for the most unstable linear wave (instead of the nonlinear simulation) gives similar results. However, the difference between the latitude of the linear waves and the nonlinear eddies varies systematically with temperature gradients. This introduces some dependence on the temperature gradient in the relationship between the linearly most unstable wavenumber and the energy-containing wavenumber.

Some have suggested that the conversion from eddy available potential energy to eddy kinetic energy is a 

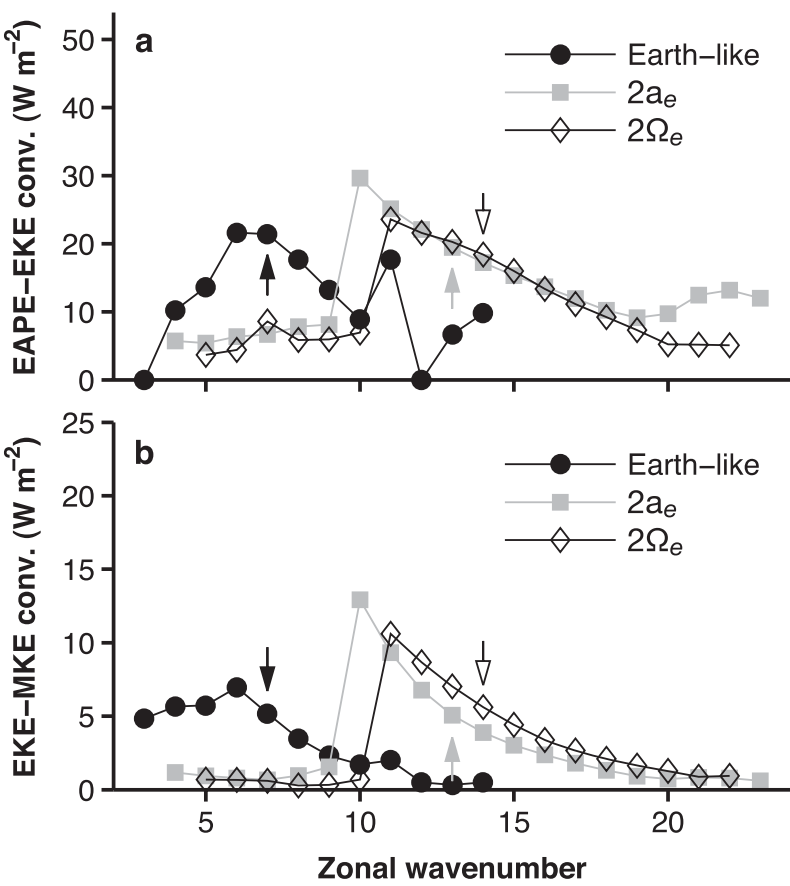

FIG. 4. Conversion of (a) eddy potential energy to eddy kinetic energy vs wavenumber and (b) eddy kinetic energy to mean kinetic energy vs wavenumber. These are the same linear waves as in Fig. 1 with arrows indicating the wavenumber with largest growth rate. The energy conversions are calculated for equal amplitude waves (i.e., the growth rate dependence has been removed).

more relevant metric for equilibrated eddies than growth rates because waves that convert more available energy to kinetic energy may attain greater amplitudes (e.g., Schneider 1981; Smith 2007). Changing to an energy conversion metric moves the dominant eddy scale to somewhat larger lengths for some of the mean flows (Fig. 4). ${ }^{2}$ The wavenumber of maximum energy conversion is typically $10 \%-20 \%$ smaller than the linearly most unstable wavenumber. This shifts the linear wavenumber closer to the nonlinear energy-containing wavenumber, although there is still at least a $20 \%$ difference.

The conversion of eddy kinetic energy to mean kinetic energy (a "sink" of eddy energy) should be considered for analogous reasons: the waves that lose more energy to the mean flow may not achieve as large an amplitude as those that transfer less. Energy conversion versus wavenumber curves have similar shapes for both eddy potential to eddy kinetic energy (Fig. 4a) and eddy

\footnotetext{
${ }^{2}$ The energy conversions in Fig. 4 are calculated using standard definitions (e.g., Lorenz 1955; Peixoto and Oort 1992). The conversion between eddy and mean kinetic energy owing to the vertical eddy flux of meridional momentum $\left(\overline{\omega^{\prime} v^{\prime}} \partial_{p} \bar{v}\right)$ is omitted, consistent with quasigeostrophic scaling.
}

to mean kinetic energy (Fig. 4b). The reason for this commonality is that both curves are controlled by the relative height and meridional scales of the waves. These two terms do not close the energy budget (note the different axis scales in Fig. 4) as there is small-scale dissipation and, more importantly, the eddy kinetic energy is growing exponentially in time.

The meridional length scales of the linear waves are of the same magnitude as the zonal length scales. Using meridional wavenumbers defined by $n_{e}-m$ (e.g., Shepherd 1987b), where $n_{e}$ is the energy-containing spherical wavenumber of the linear waves determined as in SW06 and $m$ is their zonal wavenumber, the meridional wavenumber is usually within a factor of 2 of the zonal wavenumber. However, the zonal and meridional wavenumbers do not necessarily vary together. In the nonlinear simulations, the meridional wavenumber asymptotically approaches $\sim 3$, while the zonal wavenumber continuously decreases with increasing temperature gradients (Fig. 3). This supports the interpretation of SW06 that the Rossby wavenumber becomes smaller than the spherical energy-containing wavenumber because the domain size limits the meridional eddy length scale.

\section{Eddy time scales}

The Eady growth rate,

$$
\sigma \sim(f / N) \partial_{z} \bar{u},
$$

is a time scale that emerges in quasigeostrophic theories of baroclinic instability such as the Eady and Charney problems (Lindzen and Farrell 1980; Vallis 2006). An alternative mean-field estimate for eddy time scales is proportional to the square root of mean available potential energy (MAPE), which scales like the Eady growth rate (e.g., O'Gorman and Schneider 2008a). This connects the time scale of linear waves to the eddy kinetic energy in the nonlinear simulations, in which the eddy kinetic energy is similar to (dry) MAPE over a wide range of both moist and dry climates (SW08; O'Gorman and Schneider 2008a).

We compare the Eady growth rate averaged over the latitudes of the most unstable wave (determined by where the potential temperature flux is within $70 \%$ of its maximum) and over a near-surface layer (between $\sigma=0.8$ and 0.7 ) to the growth rate of the linear waves in Fig. 5. The series of simulations with varying rotation rate and radius (Fig. 5, left) and constant relaxation time (Fig. 5, right) have compact scaling relations. The series of simulations with varying convective lapse rates have significant scatter, although for a given value of $\gamma$ there is generally a monotonic relation between the mean-field 

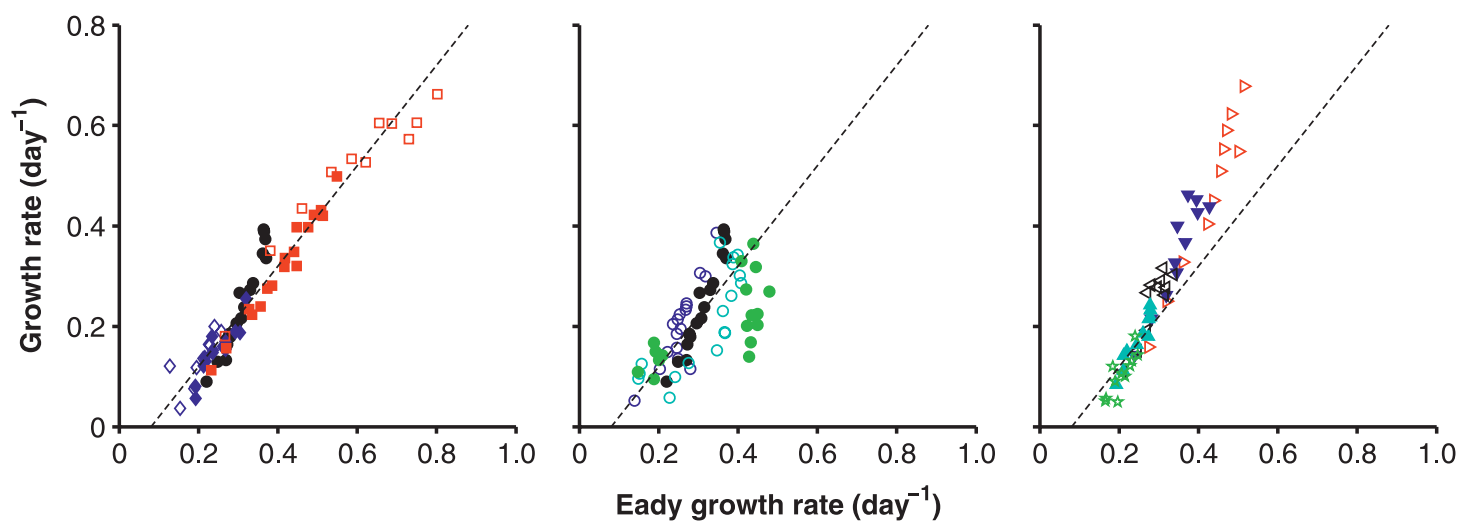

FIG. 5. Linearly most unstable growth rate vs Eady growth rate. The Eady growth rate is scaled by 0.16, a factor determined by least squares regression of all simulations. The dashed line has intercept -0.08 . Plotting symbols as in Fig. 3 .

estimate and the actual growth rate of the most unstable wave. Evaluating the Eady growth rate between $\sigma=0.7$ and $\sigma=0.6$ does not significantly change the results in Fig. 5.

The actual growth rate tends to zero and the mean flows may become baroclinically neutral at low temperature gradients; however, the Eady growth rates remain finite, which causes a nonorigin intercept of the regression lines between the actual and Eady growth rates. There are several possibilities why this occurs. First, the relationship between the Eady and actual growth rates may not be linear in the weakly unstable limit, or the actual growth rate depends on boundary layer damping (Fig. B1), whereas the Eady growth rate does not. Second, our method of calculating linear waves by integrating for a fixed time may fail to capture waves that have small but nonnegative growth rates. Third, the GCM's vertical discretization of the primitive equations is equivalent to replacing the continuous dependence on the vertical coordinate with a discrete set of interacting layers. This may introduce a spurious nonzero critical shear for instability, as in, for example, the two-layer model (e.g., Vallis 2006). Fourth, the zonal-mean flow may undergo time variations such that it is linearly unstable at certain times but neutral at others. A final possibility is that nonmodal eddy growth is an important mechanism for generating transient eddies in simulations with weak temperature gradients.

\section{Eddy energies}

The eddy kinetic energy (EKE) of the most unstable waves is more meridionally confined than that of the nonlinear eddies (Fig. 6), as was the case for the eddy fluxes in Fig. 2. The secondary maximum of EKE near the surface is another difference between the linear waves and nonlinear eddies. Because the linear calcu- lations include boundary layer damping of the same magnitude as the nonlinear simulations, the near-surface maxima of EKE of the linear waves are not directly the result of missing damping of the waves; rather, they are indicative of nonlinear modifications of the eddies in the nonlinear simulations, as has been noted by earlier investigators (Gall 1976a; Simmons and Hoskins 1976).

The EKE structure of linear waves changes compared to the most unstable wave (Fig. 6) for waves with different wavenumbers. At higher wavenumbers (shorter wavelengths), the upper tropospheric EKE maximum disappears as the vertical extent of the waves shrinks until they are confined to a shallow layer just above the surface (Held 1978). At lower wavenumbers (longer wavelengths), the structure is generally similar to the most unstable wave with some meridional broadening and deepening in the vertical. Similar changes occur for eddy potential temperature fluxes and for eddy momentum fluxes.

The partitioning between forms of eddy energies provides a way of determining the degree to which nonlinear interactions are energetically important: in the presence of an inverse cascade of eddy energy, baroclinic EKE is much smaller than the barotropic EKE and eddy available potential energy (EAPE) (Held and Larichev 1996). For the set of simulations examined here, SW08 showed that both barotropic and baroclinic eddy kinetic energies are equipartitioned with the eddy available potential energy (i.e., they all scale linearly with each other). Here, we examine the same partitionings for linear waves in the absence of nonlinear interactions.

For the linearly most unstable waves, Fig. 7 shows the partitionings between barotropic EKE $\left(\mathrm{EKE}_{\mathrm{bt}}\right)$ and EAPE, on the one hand, and between baroclinic EKE $\left(\mathrm{EKE}_{\mathrm{bc}}\right)$ and EAPE, on the other hand. The corresponding plots for the nonlinear simulations appear in 

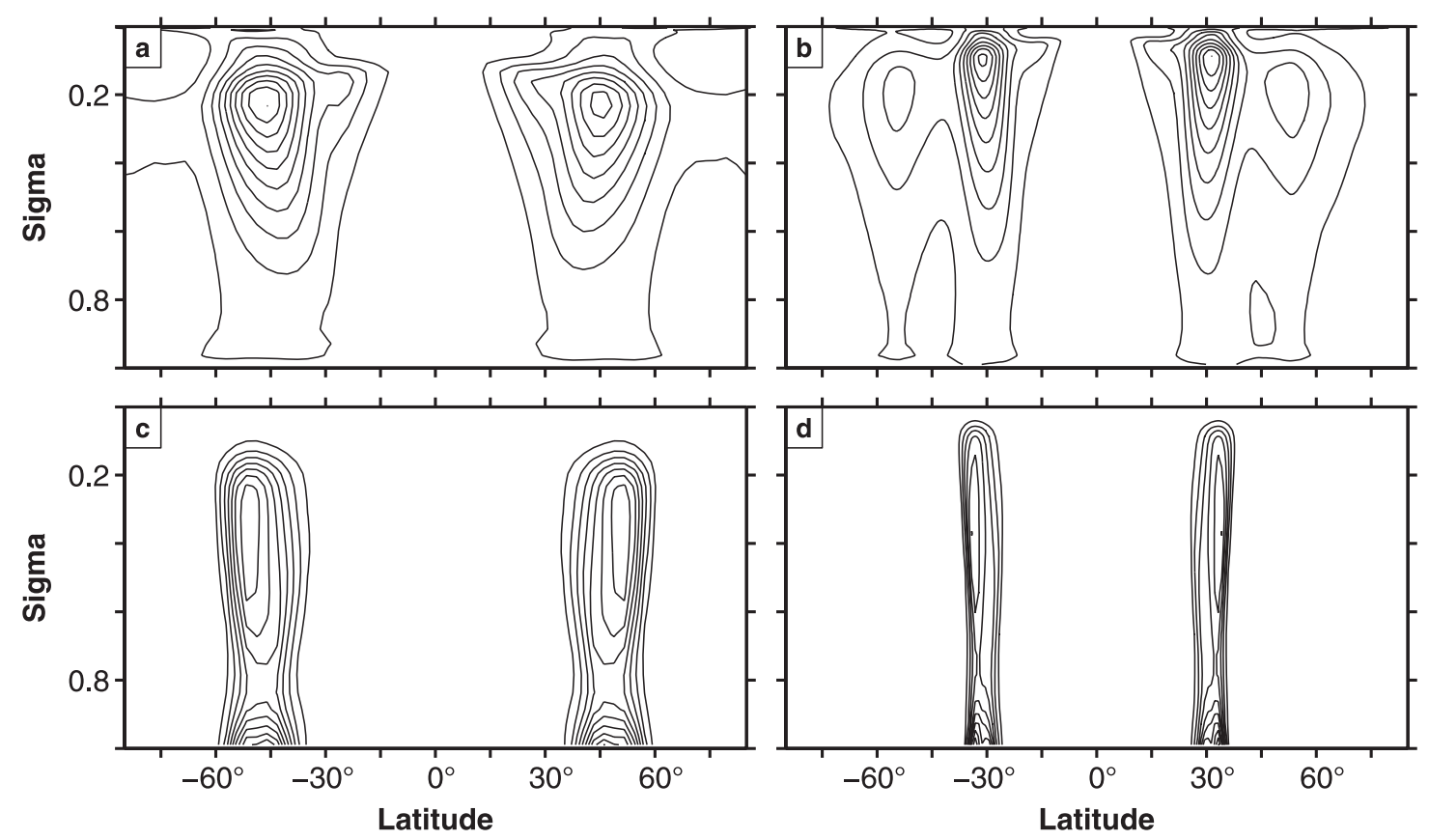

FIG. 6. Eddy kinetic energy of (a),(b) nonlinear simulations and (c),(d) the linearly most unstable wave for the same simulations as in Fig. 2. Contour interval is $10 \%$ of the maximum.

SW08 (their Figs. 5 and 6). As in the nonlinear case, the most unstable waves have equipartitioned eddy energies. The constants of proportionality in the figures here are the same as well (EAPE/EKE $E_{\mathrm{bt}} \approx 1$ and EAPE/ $\left.\mathrm{EKE}_{\mathrm{bc}} \approx 2.25\right)$, suggesting that nonlinear interactions do not significantly modify the energy partitioning of eddies. Some of the deviations from this linear scaling (e.g., the linear waves for $a=4 a_{e}$ and $\gamma=0.9$ that have $\left.\mathrm{EAPE} / \mathrm{EKE}_{\mathrm{bt}}>1\right)$ are due to mean flows with multiple jets that have linearly most unstable waves with maximum amplitude at higher-latitude jets (e.g., the most unstable waves are not located in the baroclinic zone closest to the equator); this jet dependence of energy partitionings also occurs in nonlinear simulations (O'Gorman and Schneider 2008b, their Fig. 13).

Examining the variations of these relations for linear waves of different wavenumbers (Fig. 8) shows that there is a range of $O(1)$ ratios of eddy energies. These variations are consistent with the different wave structures at different wavelengths described above, so the constants of proportionality are not a general property of linear waves. However, they may be universal for the most unstable waves: the ratios for the most unstable waves (marked by arrows in Fig. 8) are similar to the ratios for the nonlinear simulations (marked by the dashed line in Fig. 8).

Thus, the energy partitioning coefficients of the eddies in the nonlinear simulations can approximately be accounted for by those of the linearly most unstable waves. The approximate equality of the energy partitioning coefficients for the linearly most unstable waves and nonlinear eddies demonstrates that nonlinear modifications of eddies are not essential for the energetics of eddies. In particular, they are further evidence that no substantial inverse cascade of eddy energy is occurring in the nonlinear simulations. However, the linear waves do not reveal clear dependences on radius and rotation rate for the ratio of baroclinic eddy kinetic energy to eddy available potential energy, such as were seen in the nonlinear simulations (SW08).

\section{Conclusions}

We have presented the results of linear stability analyses for most of the mean flows of the simulations in SW06, SW08, and SMER, excluding a few simulations with low temperature gradients and small or possibly zero growth rates of baroclinic instability. The analyses demonstrate that the mean flows are generally baroclinically unstable and are not in a state of baroclinic adjustment.

The zonal length scale of the linearly most unstable waves is similar to the Rossby radius. The zonal length scale of the dominant linear waves is close to but somewhat smaller than (between $40 \%$ and $20 \%$, depending on ordering conventions of linear waves) the zonal length scale of the energy-containing eddies in the corresponding nonlinear simulations. Their growth 

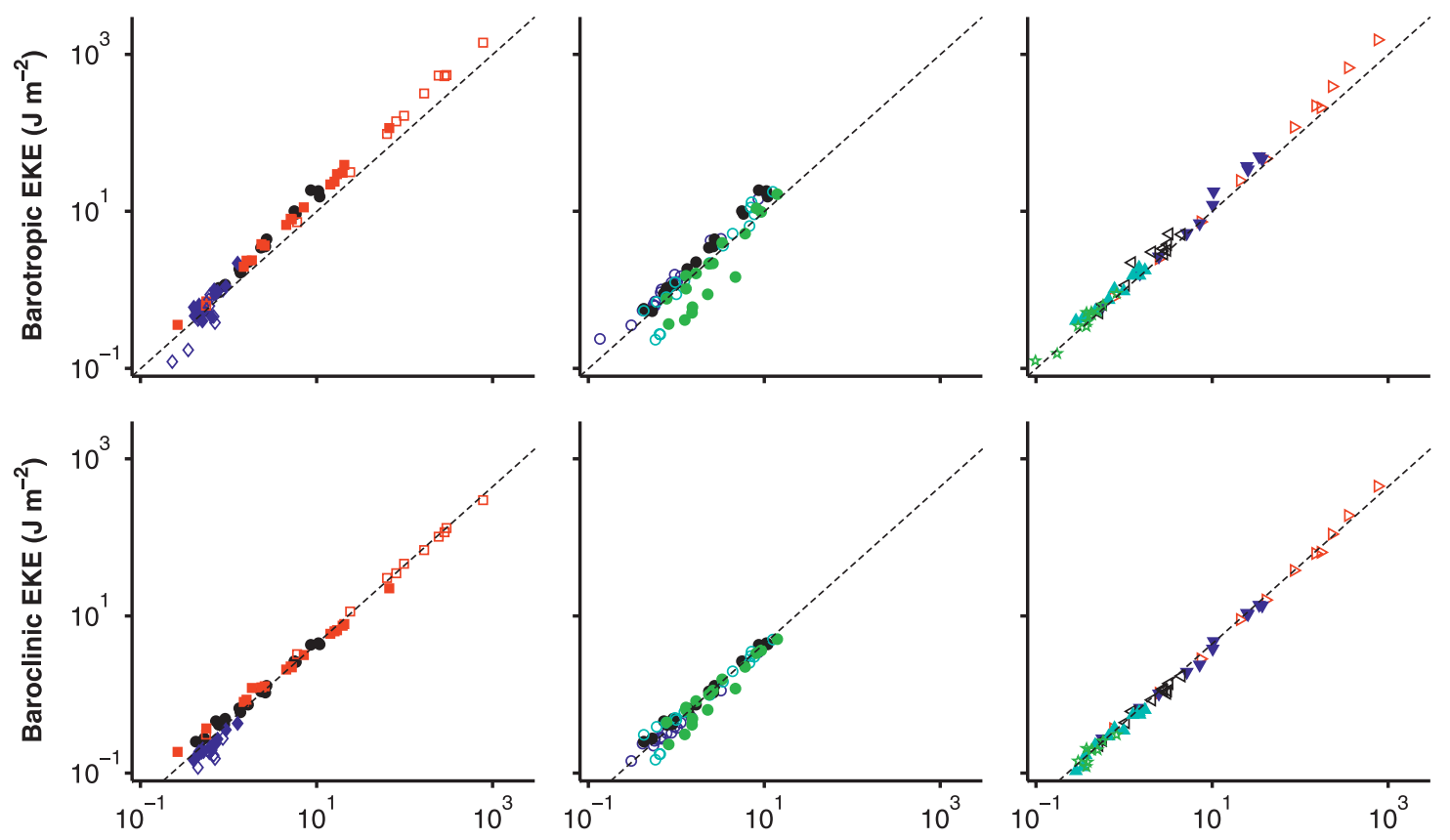

Eddy Available Potential Energy $\left(\mathrm{J} \mathrm{m}^{-2}\right)$

FIG. 7. (top) $\mathrm{EKE}_{\mathrm{bt}} \mathrm{vs}$ EAPE for the linearly most unstable wave. Dashed line is $\mathrm{EKE}_{\mathrm{bt}}=\mathrm{EAPE}$. (bottom) EKE $\mathrm{bc}_{\mathrm{bc}} \mathrm{VAPE}$ for the linearly most unstable wave. Dashed line is $2.25 \mathrm{EKE}_{\mathrm{bc}}=$ EAPE. Plotting symbols as in Fig. 3.

rates are similar to the Eady growth rate, but the scaling is not generally linear and may have a dependence on external parameters. The agreement between scales of linear waves and mean-field estimates of these scales demonstrates that the mean-field estimates based on idealized quasigeostrophic models can be used for a wide range of complex flows in a primitive equation model. The partitionings among eddy available potential energy and barotropic and baroclinic eddy kinetic energies in linear waves are also similar to those in the nonlinear eddies. Confirming the analysis in SW06, these results show that there is no substantial inverse cascade of eddy energy over a range of dry atmospheric circulations that vary from weakly to strongly unstable and include simulations with multiple jets in each hemisphere. However, the differences between linear waves and nonlinear eddies that do exist point to aspects of atmospheric macroturbulence that are modified by (weak) nonlinear eddy-eddy interactions. Particularly notable among those are modifications of the meridional scales of nonlinear eddies and of the structure of second-order quantities such as eddy momentum and heat fluxes-as shown, for a more limited set of mean flows, by Simmons and Hoskins (1978) and others.

For simple (energy balance) climate models in which the effects of large-scale eddies are not resolved explicitly but are represented parametrically, the results

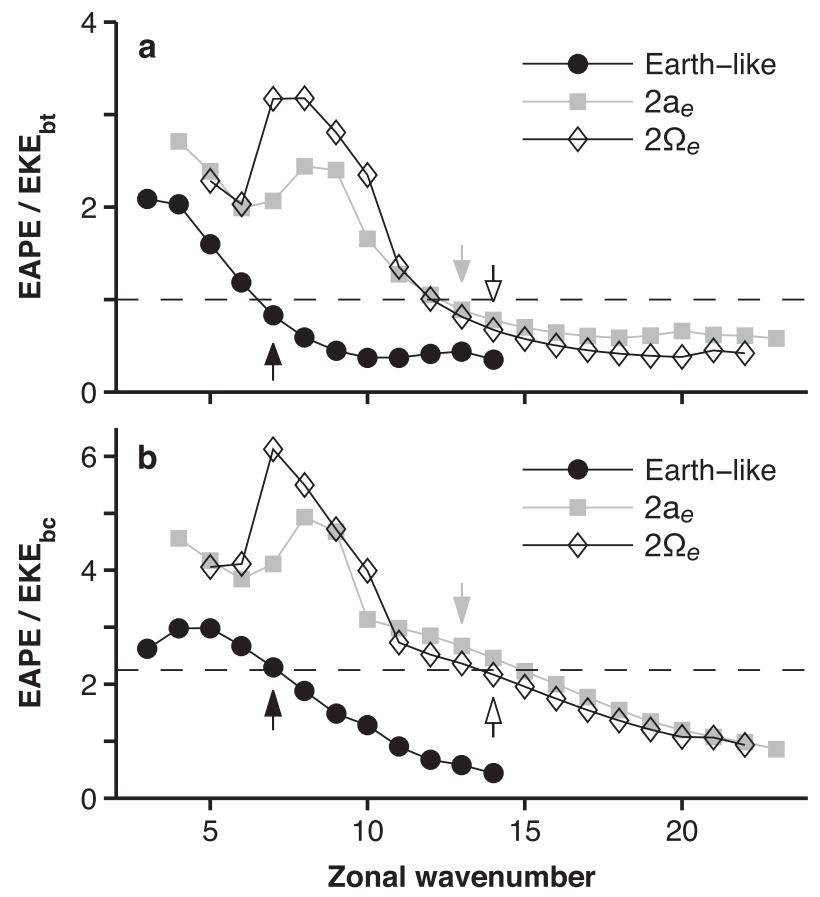

FIG. 8. Ratio of eddy energies for linear waves of different wavenumbers for the same mean flows as in Fig. 1. (a) EAPE over barotropic EKE, with dashed line EAPE $=\mathrm{EKE}_{\mathrm{bt}}$ as in top row of Fig. 7. (b) EAPE over baroclinic EKE, with dashed line EAPE = $2.25 \mathrm{EKE}_{\mathrm{bc}}$ as in bottom row of Fig. 7. Arrows indicate the wavenumber with the largest growth rate. 
here, together with those in SW06, SW08, and SMER, imply that linear stability calculations can give approximations of zonal length scales and partitionings among eddy energies. However, it will be necessary to find a way to represent weak nonlinear eddy-eddy interactions in such models to obtain accurate representations of the structure of second-order quantities such as eddy fluxes.

Acknowledgments. We thank Chris Walker for performing most of the nonlinear simulations and Simona Bordoni and Paul O'Gorman for helpful comments and discussions. T. M. Merlis is supported by a National Defense Science and Engineering Graduate Fellowship. The linear stability analyses were performed on Caltech's Division of Geological and Planetary Sciences Dell cluster. The program code for the simulations, based on the Flexible Modeling System of the Geophysical Fluid Dynamics Laboratory, and the simulation results themselves are available from the authors upon request.

\section{APPENDIX A}

\section{Determination of Growth Rate}

For each of the prognostic variables (vorticity $\zeta$, divergence $\delta$, temperature, and the logarithm of surface pressure), the root mean square of the perturbed zonal spectral coefficients averaged over levels ([ ]) and meridional (\{\}) dimensions is calculated:

$$
\Psi_{\mathrm{rms}}=\sqrt{\left\{\left[\operatorname{Re}(\hat{\Psi})^{2}+\operatorname{Im}(\hat{\Psi})^{2}\right]\right\}} .
$$

The four prognostic variables are summed with the following weights to account for their different magnitudes:

$$
Q=\frac{\zeta_{\mathrm{rms}}}{\zeta_{0}}+\frac{\delta_{\mathrm{rms}}}{\delta_{0}}+\frac{T_{\mathrm{rms}}}{T_{0}}+\frac{\left(\log p_{s}\right)_{\mathrm{rms}}}{\log p_{0}}
$$

with $\zeta_{0}=10^{-5} \mathrm{~s}^{-1}, \delta_{0}=10^{-5} \mathrm{~s}^{-1}, T_{0}=1 \mathrm{~K}$, and $\log p_{0}=300 \mathrm{~Pa}$.

The growth rate is then

$$
r=\frac{1}{\Delta t} \log \left[\frac{Q(t)}{Q(t-\Delta t)}\right],
$$

where $\Delta t=1$ day (using 2 days or 0.5 days gives similar results). Other ways of determining the growth rate (e.g., Simmons and Hoskins 1976; Hall and Sardeshmukh 1998) give similar results. The daily growth rate is av- eraged over the last 6 days of the linear stability calculation.

We found that some linear waves had oscillatory growth rates. This typically occurred for short wavelengths (large wavenumbers), and, as a result, generally these had growth rates well below those of the most unstable waves. In a handful of cases, the maximum growth rate attained over the course of the oscillation would be larger than the (steady) growth rate of the most unstable wave. For this reason, we use the growth rate averaged over 6 days instead of the growth rate averaged over 1 day. This choice changes the most unstable wavenumber in less than $5 \%$ of the mean flows we analyzed, so none of the results or conclusions depends on it. It is unclear what causes the oscillatory growth rates. However, after sufficient time ( $\sim 200$ days in some cases), the oscillations decay and leave a steady growth rate.

\section{APPENDIX B}

\section{Details of the Linear GCM}

We linearize the GFDL spectral dynamical core about the time, zonal, and hemispheric mean of the last $(100,25,10)$ days of the horizontal resolution (T42, T85, T127) simulations in SW06, SW08, and SMER. The dynamical core of the GCM is modified in two ways to ensure linearity. Following Simmons and Hoskins (1976), only the spectral coefficients of the perturbed zonal wavenumber are advanced forward in time, and the growing wave is kept at small amplitude by rescaling. The rescaling is performed when the maximum surface pressure deviation grows to $10 \mathrm{~Pa}$, at which point it is reduced to $1 \mathrm{~Pa}$; many alternative rescaling criteria are possible and give similar results. The baroclinic wave is initialized by perturbing the odd meridional spectral coefficients of vorticity between wavenumbers 3 and 39 equally at all model levels, which ensures hemispheric symmetry. Time is discretized using a second-order leapfrog method, so the perturbation is added to both initial time steps. The initial perturbation is not geostrophically balanced, but the model is integrated for sufficient time to ensure geostrophic adjustment has occurred by the time quantities are measured. The linear model is run for 33 days, at which point waves with typical growth rates have doubled in amplitude at least three times. Because the model is run for a fixed number of days independent of growth rate, more slowly growing waves may not be as accurately computed; however, experiments run for 58 days show little difference in structure or growth rate ("long" in Fig. B1). 

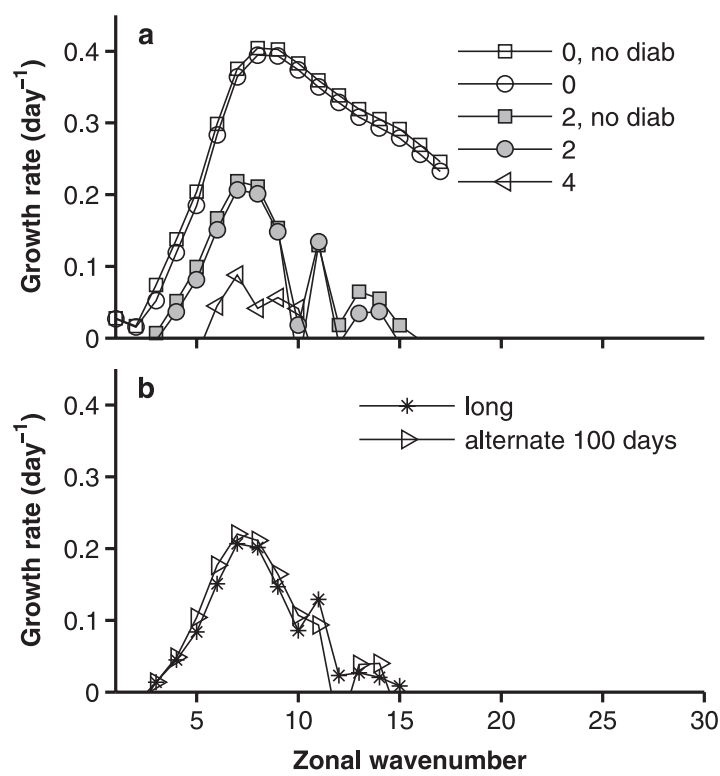

FIG. B1. (a) Sensitivity of growth rates to Rayleigh drag and Newtonian relaxation. Numbers in legend refer to damping coefficient in units day ${ }^{-1}$ (e.g., 0 day $^{-1}$ has no boundary layer damping), and "no diab" has a much larger Newtonian relaxation time scale. (b) A "long" linear stability analysis, which was integrated for 58 instead of 33 days, and a linear stability analysis of a different 100-day average mean flow of the same nonlinear simulation.

To determine how sensitive the linear waves are to the mechanical and diabatic forcing, we performed additional experiments with Earth-like mean flow (Fig. 2a) in which we increased and decreased the boundary layer damping time scale and greatly reduced the diabatic forcing by increasing the relaxation time scale to $1.2 \times$ $10^{4}$ days uniformly in latitude and pressure. The sensitivity to boundary layer Rayleigh drag and Newtonian cooling is similar to that in Valdes and Hoskins (1988): mechanical damping in the boundary layer neutralizes high-wavenumber instability, and there is little sensitivity to thermal forcing, consistent with Newtonian relaxation time scales generally being long compared with eddy time scales (Fig. B1). We have also reduced the diabatic forcing for a linear stability calculation with the shortest relaxation time in the corresponding nonlinear simulations ( $\tau=12.5$ days); there was little sensitivity even in this strongly forced case.

We have performed additional nonlinear simulations in which the turbulent boundary layer scheme of the GCM was replaced with Rayleigh drag and have performed stability analyses of the resulting mean flows. In this case, where both the thermal and mechanical damping of the nonlinear and linear cases are identical, the mean flows are also generally baroclinically unstable. In linear stability analyses with small Rayleigh drag throughout the atmospheric column, as in Hall and Sardeshmukh (1998), the instability likewise is not neutralized, and the most unstable wavenumber is not significantly affected by the added drag.

\section{APPENDIX C}

\section{Definitions of Eddy Scales}

\section{a. Energy-containing scale}

The energy-containing wavenumber is estimated using a -2 moment of the zonal spectrum of eddy meridional kinetic energy,

$$
m_{e}^{2}=\frac{\sum_{m} E_{m}}{\sum_{m} m^{-2} E_{m}},
$$

evaluated at the latitude of maximum eddy potential temperature flux. This choice gives wavenumbers that are close to the maximum of the spectrum. An alternative definition that is commonly used,

$$
m_{e}=\frac{\sum_{m} m E_{m}}{\sum_{m} E_{m}},
$$

gives energy-containing wavenumbers that are larger than the maximum of the spectrum or the -2 moment because the spectrum of eddy meridional kinetic energy is asymmetric [see Frierson et al. (2006, their Fig. 14a) for a representative example]. This definition would lead to somewhat closer agreement with the linearly most unstable wavenumber $\left(m_{e} \sim 0.8 m_{l}\right)$.

\section{b. Rossby radius}

The Rossby radius is computed using near-surface quantities:

$$
L_{R}=c_{R} \frac{N_{p}\left(\bar{p}_{s}-\bar{p}_{t}\right)}{f} .
$$

The zonal Rossby wavenumber is $m_{R}=a \cos \left(\phi_{\mathrm{ref}}\right) / L_{R}$, where $\phi_{\text {ref }}$ is the latitude of maximum eddy potential temperature flux, as in the definition of energy-containing wavenumber. The constant $c_{R}=0.8$ is chosen so that the Rossby wavenumbers are close to the wavenumbers of the most unstable waves. Including a supercriticality factor as in SW08 or in SW06 does not change Fig. 3 significantly.

\section{REFERENCES}

Edmon, H., B. Hoskins, and M. McIntyre, 1980: Eliassen-Palm cross sections for the troposphere. J. Atmos. Sci., 37, 2600-2616. 
Frierson, D., I. Held, and P. Zurita-Gotor, 2006: A gray-radiation aquaplanet moist GCM. Part I: Static stability and eddy scale. J. Atmos. Sci., 63, 2548-2566.

Gall, R., 1976a: A comparison of linear baroclinic instability theory with the eddy statistics of a general circulation model. J. Atmos. Sci., 33, 349-373.

_ 1976b: Structural changes of growing baroclinic waves. J. Atmos. Sci., 33, 374-390.

Hall, N., and P. Sardeshmukh, 1998: Is the time-mean Northern Hemisphere flow baroclinically unstable? J. Atmos. Sci., 55, $41-56$

Held, I., 1978: The vertical scale of an unstable baroclinic wave and its importance for eddy heat flux parameterizations. J. Atmos. Sci., 35, 572-576.

_ 1999: The macroturbulence of the troposphere. Tellus, 51A-B, 59-70.

— , and M. Suarez, 1994: A proposal for the intercomparison of the dynamical cores of atmospheric general circulation models. Bull. Amer. Meteor. Soc., 75, 1825-1830.

_ mogeneous, baroclinically unstable flow on a beta plane. J. Atmos. Sci., 53, 946-952.

Lindzen, R., and B. Farrell, 1980: A simple approximate result for the maximum growth rate of baroclinic instabilities. J. Atmos. Sci., 37, 1648-1654.

Lorenz, E., 1955: Available potential energy and the maintenance of the general circulation. Tellus, 7, 157-167.

O'Gorman, P., and T. Schneider, 2007: Recovery of atmospheric flow statistics in a general circulation model of the atmosphere without nonlinear eddy-eddy interactions. Geophys. Res. Lett., 34, L22801, doi:10.1029/2007GL031779.

— idealized simulations of changed climates. J. Climate, 21, $5797-5806$.

- and _ 2008b: Weather-layer dynamics of baroclinic eddies and multiple jets in an idealized general circulation model. $J$. Atmos. Sci., 65, 524-535.
Peixoto, J., and A. Oort, 1992: Physics of Climate. American Institute of Physics, 520 pp.

Schneider, E., 1981: On the amplitudes reached by baroclinically unstable disturbances. J. Atmos. Sci., 38, 2142-2149.

Schneider, T., 2004: The tropopause and the thermal stratification in the extratropics of a dry atmosphere. J. Atmos. Sci., 61, $1317-1340$.

- and C. Walker, 2006: Self-organization of atmospheric macroturbulence into critical states of weak nonlinear eddyeddy interactions. J. Atmos. Sci., 63, 1569-1586.

—, and —, 2008: Scaling laws and regime transitions of macroturbulence in dry atmospheres. J. Atmos. Sci., 65, 2153-2173.

Shepherd, T., 1987a: Rossby waves and two-dimensional turbulence in a large-scale zonal jet. J. Fluid Mech., 183, 467-509.

_ 1987b: A spectral view of nonlinear fluxes and stationarytransient interaction in the atmosphere. J. Atmos. Sci., 44, 1166-1179.

Simmons, A., and B. Hoskins, 1976: Baroclinic instability on the sphere: Normal modes of the primitive and quasi-geostrophic equations. J. Atmos. Sci., 33, 1454-1477.

$\longrightarrow$, and - 1978: The life cycles of some nonlinear baroclinic waves. J. Atmos. Sci., 35, 414-432.

Smagorinsky, J., S. Manabe, and J. Holloway, 1965: Numerical results from a nine-level general circulation model of the atmosphere. Mon. Wea. Rev., 93, 727-768.

Smith, K., 2007: The geography of linear baroclinic instability in Earth's oceans. J. Mar. Res., 65, 655-683.

Valdes, P., and B. Hoskins, 1988: Baroclinic instability of the zonally averaged flow with boundary layer damping. J. Atmos. Sci., 45, 1584-1593.

Vallis, G., 2006: Atmospheric and Oceanic Fluid Dynamics: Fundamentals and Large-Scale Circulation. Cambridge University Press, $771 \mathrm{pp}$

Walker, C. C., and T. Schneider, 2006: Eddy influences on Hadley circulations: Simulations with an idealized GCM. J. Atmos. Sci., 63, 3333-3350. 